\title{
Quatre disques de musique portugaise
}

\section{Anne Caufriez}

\section{OpenEdition \\ Journals}

Édition électronique

URL : http://journals.openedition.org/ethnomusicologie/2410

ISSN : 2235-7688

Éditeur

ADEM - Ateliers d'ethnomusicologie

Édition imprimée

Date de publication : 1 janvier 1990

Pagination : 239-248

ISBN : 2-8257-0423-7

ISSN : $1662-372 X$

Référence électronique

Anne Caufriez, "Quatre disques de musique portugaise », Cahiers d'ethnomusicologie [En ligne],

3| 1990, mis en ligne le 15 octobre 2011, consulté le 19 avril 2019. URL : http://

journals.openedition.org/ethnomusicologie/2410

Ce document a été généré automatiquement le 19 avril 2019.

Article L.111-1 du Code de la propriété intellectuelle. 


\title{
Quatre disques de musique portugaise
}

\author{
Anne Caufriez
}

\section{RÉFÉRENCE}

Portugal - Musiques traditionnelles de l'Alentejo. Enregistrements de Roselyne François et Manuel Gomes. Un CD Playa Sound PS 65017, 1987.

Portugal - Musique traditionnelle du Portugal. Enregistrements de Hubert de Fraysseix et Virgilio Pereira. Un CD Auvidis D 8008, 1988 [Collection UNESCO « Musique et musiciens du monde »].

Portugal - Fernando Machado Soares, le Fado de Coimbra. Un CD Ocora/Radio France C 559041, 1988.

Fado... fados - La célèbre Fernando Maria - Maria-Gloria Guedes - Lucinda Sobral - Helder Antonio. Un CD Arion ARN 64072, 1989.

Portugal - Musiques traditionnelles de l'Alentejo. Enregistrements de Roselyne François et Manuel Gomes. Un CD Playa Sound PS 65017, 1987.

Portugal - Musique traditionnelle du Portugal. Enregistrements de Hubert de Fraysseix et Virgilio Pereira. Un CD Auvidis D 8008, 1988 [Collection UNESCO « Musique et musiciens du monde »].

Portugal - Fernando Machado Soares, le Fado de Coimbra. Un CD Ocora/Radio France C 559041, 1988.

Fado... fados - La célèbre Fernando Maria - Maria-Gloria Guedes - Lucinda Sobral - Helder Antonio. Un CD Arion ARN 64072, 1989.

Le premier disque, édité par Playa Sound, porte exclusivement sur l'Alentejo, province située au sud du pays et connue pour ses polyphonies masculines moulées sur le collectif agricole. Dans une région de grands propriétaires fonciers, l'importance légitime de ces chœurs d'hommes sur le plan de la lutte politique a oblitéré pendant longtemps l'intérêt que peut y présenter la musique instrumentale. Celle-ci se manifeste notamment par l'ensemble flûte-tambour, par la flûte traversière, le tambourin, le tambour à friction et la viola campaniça, guère étudiée jusqu'ici. 
2 Il s'agit d'une guitare généralement munie de quatre doubles cordes (parfois plus) qui a ses équivalents dans d'autres régions du Portugal comme la Beira ou les îles des Açores et de Madère. Sur le plan du timbre, c'est aussi une des plus jolies guitares campagnardes du pays. Son existence en Alentejo a été récemment redécouverte par un avocat, J. A. Sardinha, grand amateur de musique traditionnelle, qui a prospecté plusieurs provinces du Portugal. Le disque qu'il a consacré à la viola campaniça de l'Alentejo $(1986)^{1}$ a fait sortir de l'oubli cet instrument qu'on croyait abandonné pour révéler son succès présent dans une partie de la province. Cette guitare accompagne notamment la danse, ainsi que des chants de divertissement souvent polyphoniques.

3 C'est aussi cet instrument que privilégie le disque Playa Sound dont les enregistrements sont signés par Roselyne François et Manuel Gomes. Mais il permet également d'écouter une très jolie flûte champêtre (plages $5,14,22,30$ ) et les chœurs d'ouvriers agricoles tels qu'ils se pratiquent aujourd'hui $(1,11,19,28,32)$.

Ce qui frappe d'emblée l'auditeur, c'est la brièveté des plages, au nombre de 32 ; certaines d'entre elles n'atteignent même pas une minute de durée. C'est un chiffre record pour la musique populaire européenne. Et quelle frustation pour l'auditeur ! Ce choix pourrait correspondre au désir de présenter le plus large échantillonnage possible des musiques existantes. En réalité, il n'en est rien. L'infinie division des musiques ne se fait qu'au prix d'une longue répétition de répertoires semblables. Ceci concerne surtout la musique de viola campaniça qui, certes, représente un instrument villageois traditionnel intéressant mais dont les mélodies actuelles manquent parfois de séduction artistique. Que ce soit sur le disque de J. A. Sardinha ou sur celui-ci, force nous est de constater que les répertoires de cet instrument et les chants qui leur sont liés sont d'une grande monotonie. Mais tel est l'état de la tradition en Alentejo. Sans doute assiste-t-on ici à un décalage chronologique entre la tradition vocale et la tradition instrumentale, ce qui ne devrait pas nécessairement entraver le plaisir de l'oreille... Toujours est-il qu'il existe ailleurs de bien plus beaux exemples de ce type de chanson pan-portugaise, de caractère nostalgique et d'origine assez récente, tels les despiques de Madère ou du Douro Litoral. Le répertoire de la viola campaniça ne justifiait donc pas la publication d'un second disque qui, de surcroît, est la réplique médiocre du premier. En effet, R. François et $\mathrm{M}$. Gomes ont enregistré exactement les mêmes guitaristes que J. A. Sardinha, et de plus dans des séquences écourtées. En outre, si ce dernier a su capter les musiciens au meilleur de leur inspiration, les auteurs du disque Playa Sound les ont saisi dans un flux de fausses notes et d'accords grinçants. Pourtant, la comparaison montre que nous n'assistons pas à un manque d'entraînement des musiciens. Les plages 9, 10, 18 et 24 consacrées à cette vieille guitare campagnarde figurent parmi les plus audibles.

Dans ces conditions, pourquoi n'avoir pas accordé plus d'importance à la très jolie flûte que l'on entend en solo aux plages 5, 14, 22 et 30 ? C'est d'un grand flûtiste qu'émanent ces morceaux d'une exécution légère et agile, lequel fait jaillir de son instrument des timbres de toute beauté. Et on ne saurait reprocher ici la brièveté des plages, qui est propre aux mélodies de cette flûte pastorale.

Quant aux célèbres polyphonies masculines $a$ capella, seules celles des plages 19 et 28 ont retenu notre attention parce que les seules conformes aux canons de l'interprétation traditionnelle dont se dégage force et conviction. Si la prolifération de ces chœurs d'hommes à des fins de concours entre les villages a permis que le genre se ressource, elle a certainement stéréotypé ses modalités d'exécution. D'autre part, il est parfois difficile de réunir un chœur complet à cause de l'émigration des ouvriers agricoles vers Lisbonne 
et les pays étrangers. C'est ce qui semble être arrivé aux auteurs de ce disque pour les plages 1 et 11 .

Fig. 1 : Deux paysannes du Tras-os-Montes chantant la ballade

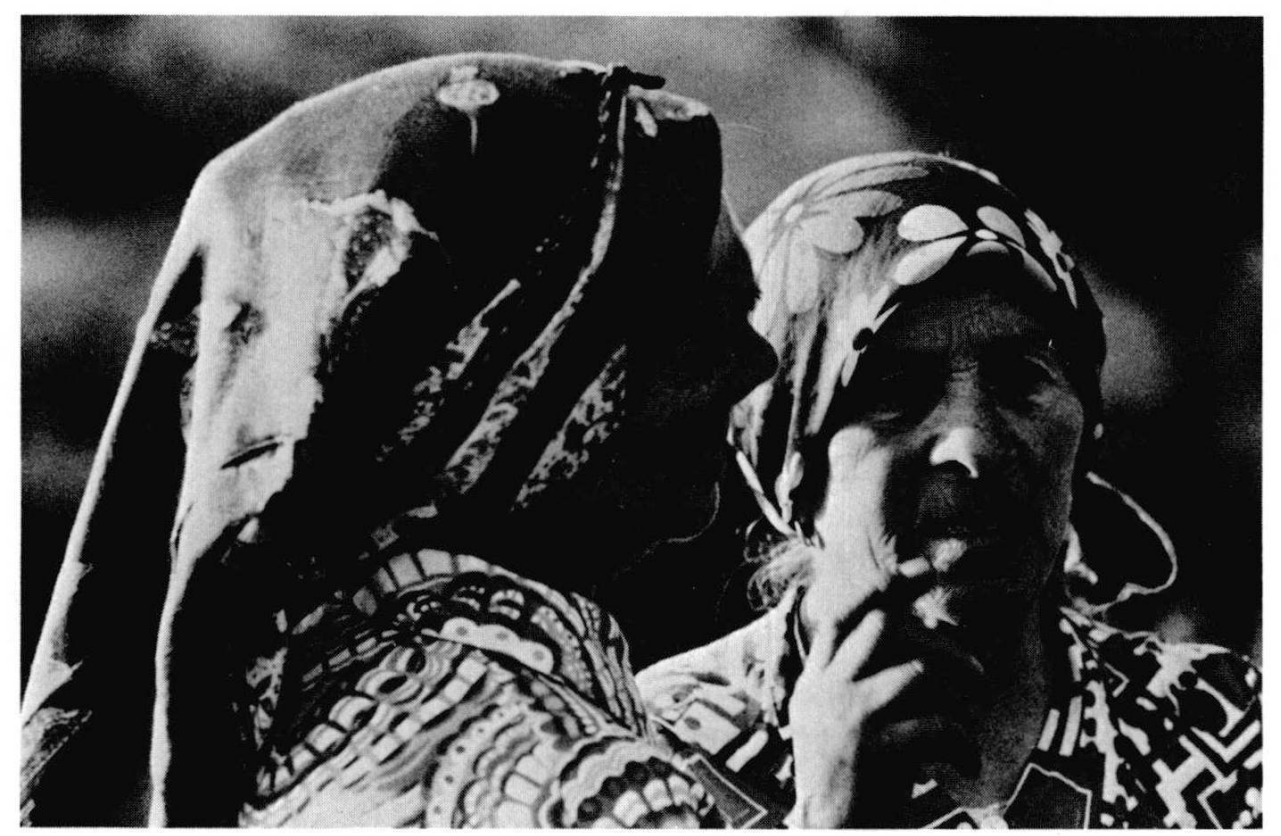

Photo : Anne Caufriez.

7 Assistons-nous à un affaiblissement de la tradition ou tout simplement à la superficialité du travail de terrain? Le texte d'introduction de la brochure révèle en tout cas une parfaite compréhension du contexte social dans lequel la musique de ce disque a été enregistrée. Il embrasse avec beaucoup d'acuité le contexte de la vie rurale en Alentejo et dresse un tableau synthétique du rôle qu'y joue la musique.

Les musiciens sont ici mentionnés et remerciés avec respect. Mais il est dommage qu'une telle profondeur de perception du milieu n'ait pas été mise à profit pour nourrir les commentaires des plages qui restent pauvres et imprécis. On aurait aussi souhaité plus d'informations originales, car quelques propos émis sur la viola campaniça proviennent directement de la brochure du disque de J. A. Sardinha, sans que soit mentionnée leur provenance.

9 En résumé, les beaux solos de la petite flûte champêtre constitueront la principale joie du mélomane. L'autre mérite de cette publication est de réactualiser les polyphonies masculines de l'Alentejo et de révéler de nouveaux répertoires pour la viola campaniça. Plutôt que le produit d'une enquête socio-musicale, ce disque est le fruit d'une convivialité paysanne, démarche qui n'induit pas nécessairement la qualité artistique des musiques recueillies.

10 Le disque Portugal publié dans la collection UNESCO en 1972 vient de faire l'objet d'une réédition en compact (1988) sous la marque française Auvidis. Il associe les enregistrements de terrain de deux auteurs différents: Hubert de Fraysseix et Virgilio Pereira $^{2}$. Il présente des musiques provenant de plusieurs provinces du Portugal : la Beira Baixa, l'Alentejo, le Douro Litoral et la région de Lisbonne. Quant aux commentaires de la brochure, ils sont signés par Alain Daniélou. Seul disque de musique traditionnelle du 
Portugal que la collection de l'UNESCO ait publié, celui-ci révèle quelques très beaux documents qui sont représentatifs de la variété des genres rencontrés dans les campagnes de ce pays ou dans les quartiers populaires de ses villes. La partie la plus remarquable de la publication est certes celle qui concerne fado, cette chanson urbaine aux accents mélodramatiques, interprétée ici par Maria Teresa de Noronha et Manuel Vicente, deux grands noms du fado de Lisbonne.

11 Né au siècle passé dans les bas-quartiers de la capitale, le fado est un genre attaché à la vie des bars et des maisons de prostitution qui en font toute l'animation nocturne. C'est dans la taverne populaire qu'il trouve sa véritable expression encore aujourd'hui, bien qu'il ait évolué partiellement vers une musique de scène. Avec M. T. Noronha, nous assistons à un fado qui se dégage de la tradition orale au profit d'une inspiration plus «cultivée » où texte et musique sont composés par des artistes connus (plages 9 et 10). Sa voix à la fois dense et contenue est de toute beauté. De par ses mouvements de retour et ses accents pathétiques, elle traduit tous les registres de la tristesse qui est l'essence même du fado. Non moins remarquables sont les deux guitaristes qui l'accompagnent, dont le jeu clair et limpide est d'une grande musicalité, rendant à la guitarra portuguesa toute la qualité de ses timbres métalliques finement découpés.

12 Quant aux fados de Manuel Vicente. ils se rattachent plutôt à des textes inspirés de la tradition orale et à des musiques recréées à chaque fois selon les besoins de l'improvisation. Ce qui caractérise l'art de ce fadiste poignant, c'est une voix d'une grande constance dramatique, sur laquelle vient s'articuler un jeu instrumental très dépouillé et très subtil, de caractère impressionniste. La "guitare portugaise » qui est l'instrument mélodique du fado procède ici par petites touches successives venant nuancer le chant de quelques pointes de nostalgie, alors que les accords de la guitare classique se font discrets.

13 Ces documents prennent d'autant plus de valeur que le fado est un genre fragile, constamment menacé par la vulgarité ou l'emphase. Trop tributaire des états d'âme qui l'inspirent, cette chanson propre au Portugal peut perdre instantanément tout son impact émotionnel. A cet égard, elle diffère du flamenco qui a des racines et des modes de transmission plus solides. Aussi, les quelques fados de ce disque sont-ils des chefs d'œuvre en leur genre, des modèles artistiques tant par la sobriété et la pureté d'interprétation qui les caractérisent que par leurs nuances mélodiques.

Pour ce qui est des musiques du terroir, notre attention a été tout particulièrement attirée par un chant féminin de la Beira Baixa qui est acompagné du tambour adufe (plage 5). Ce chant, interprété pour la Saint-Jean, appartient à cette musique plus archaïsante des vieilles airs pastorales du Portugal où les femmes se sont spécialisées dans la percussion, L'adufe soutient ici une merveilleuse mélodie de caractère modal, laquelle évolue sur un pentacorde subtilement fleuri par une voix d'une grande pureté. Tandis que les plages 1 et 3 laissent entendre des orchestres d'adufes de qualité, les mélodies que ceux-ci soutiennent sont nettement moins caractéristiques de la région.

Les orchestres de guitares traditionnelles du Douro Litoral, d'origine plus récente, représentent un des aspects les plus originaux de la facture instrumentale portugaise, précieux témoignages de guitares historiques tombées en désuétude (plage 14). Les interprètes, ici excellents, rendent à cette musique de la côte Nord ses qualités de gaieté et d'enthousiasme, puisqu'elle anime la danse. 

Carême A encommendaçao das almas d'une belle interprétation, lequel correspond à un archaïque culte populaire voué aux âmes du Purgatoire. Mais c'est surtout le chant de travail du tailleur de pierres qui mérite l'attention. Cette mélopée qui évolue sur des échelles modales est véritablement sublime par rapport à d'autres exemples connus qui font référence. Les écarts et les déplacements de cette voix très nuancée créent une modulation permanente de la mélodie, qui s'égare dans de multiples registres et dont les points de chute sont des tierces descendantes.

à part les plages dont nous venons de relever l'intérêt, ce disque présente dans son ensemble des musiques trop hétérogènes et inégales pour constituer une grande publication. De plus, pourquoi briser son unité en associant les enregistrements de deux auteurs différents dont le travail de terrain présente de grands écarts de répertoires et de chronologie? Car si H. de Fraysseix a probablement réalisé ses enregistrements dans les années 1965-70 (la première édition de ce disque remontant à 1972), on sait que feu V. Pereira effectua les siens vers les années 1940-50. Grande figure de l'ethnomusicologie nationale, ce dernier a collecté sur le terrain un éventail de musiques traditionnelles qui aurait suffi pour éditer plusieurs disques. Utiliser ses enregistrements pour combler les lacunes de celui-ci est quelque peu dépréciatif à son égard.

Bien plus que par l'inégalité de ses plages, ce disque pêche par le contenu de sa notice, que signe pourtant Alain Daniélou. Le texte introductif évoque des généralités historiques bien éloignées de l'expérience vivante de l'ethnographe. Quelques propos nostalgiques sur l'Empire romain et plusieurs clichés sur le fado viennent remplacer l'effort d'investigation des sources écrites les plus élémentaires et la volonté de pénétration des milieux où se pratique la musique. La comparaison avec les traditions musicales d'Extrême-Orient n'éclaire en rien la nature des répertoires présentés dans ce disque. Les quelques mots d'introduction au fado sont tout aussi déplacés, que ce soit au niveau historique ou à celui de la définition de la saudade, ce sentiment qui l'inspire si profondément. La saudade n'est pas « la solitude de la tristesse » comme le dit Daniélou, mais bien le regret, l'infinie nostalgie d'un être aimé, d'un événement, réel ou imaginaire. 
Fig. 2 : Un grand chanteur de ballades du Tras-os-Montes

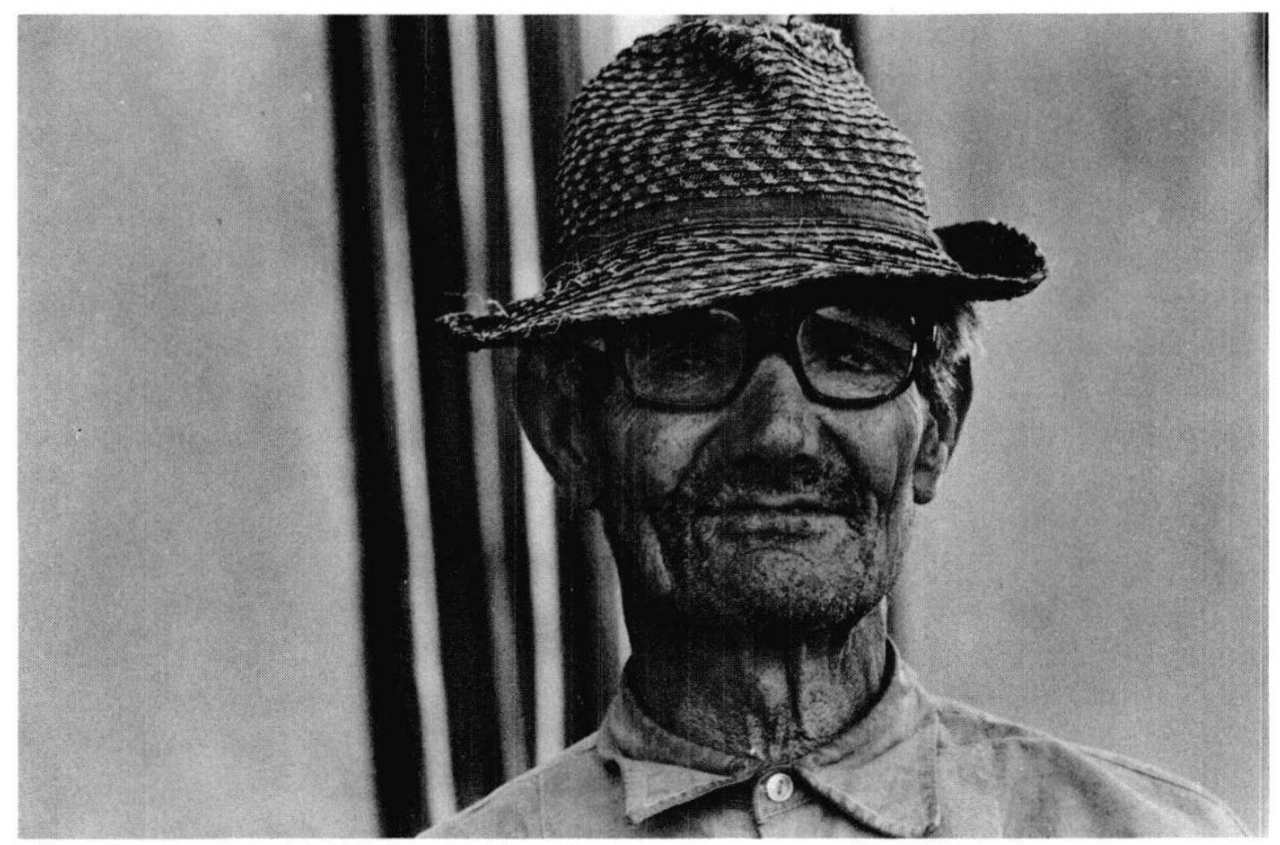

Photo : Anne Caufriez.

19 La musique de ce disque n'est-elle pas reléguée au rang de folklore dont même la fonction sociale indiffère ? En effet, pas un commentaire ne touche au cadre de l'interprétation des répertoires sélectionnés, et les textes des chants nous sont rendus de façon fragmentaire. Aucun nom de musicien n'est mentionné, pas plus que celui des villages d'où proviennent les enregistrements. En outre, la composition des orchestres et le nom vernaculaire des instruments de musique ne sont jamais précisés. Le dédain pour les artistes de ce disque est poussé à un tel point qu'aucune photo n'est accompagnée de sa légende et que la photo de couverture montre un joueur de hautbois qu'on n'entend nulle part ${ }^{3}$.

Par son caractère hétéroclite et par son commentaire approximatif, ce disque appartient à une conception périmée de la musique européenne. Édité dans une marque prestigieuse et réédité récemment, il est regrettable que le texte de la brochure n'ait fait l'objet d'aucune révision, d'autant plus qu'un effort a été apporté à la gravure. Cette publication constitue néanmoins une référence intéressante sur le Portugal traditionnel, dont elle livre quelques musiques maîtresses.

21 Lisbonne et Coimbra représentent les deux grands foyers du fado, cette chanson originale au Portugal dont elle reflète les états d'âme particuliers. Le disque Le Fado de Coimbra, publié dans la collection Ocora de Radio France (Paris 1988) est consacré au chanteur Fernando Machado Soares, représentant de cette école.

De par son contexte social comme par son style mélodique, le fado de Coimbra se rattache à une tradition très différente de celui de Lisbonne, même s'il chante aussi l'amour et s'accompagne des mêmes guitares. C'est essentiellement par les hommes qu'il est interprété, étant l'expression privilégiée des étudiants de l'Université. Ceux-ci, revêtus de grandes capes noires, chantaient souvent le fado, le soir, sous la fenêtre des jeunes filles, ou se réunissaient dans les cafés de la ville pour se divertir au son des guitares. 
Si ces coutumes tendent à disparaitre aujourd'hui, le fado, ainsi que la chanson qui s'en inspire, trouvent toujours leur foyer de création à l'Université. Le fado de Coimbra se rattache donc à une tradition plus érudite que celui de Lisbonne, bien qu'il ait connu des sources d'inspiration variées allant de la musique savante et des grands poètes à la musique populaire qui marque le fado tourné vers les causes sociales. Ce courant plus récent est notamment représenté par les chanteurs Zeca Alfonso et Adriano Correia de Oliveira, aujourd'hui disparus, que j'ai personnellement connus.

Ces artistes ont opéré un véritable retour à la culture paysanne, en interprétant notamment de vieilles ballades traditionnelles mais aussi les textes de poètes socialisants comme Manuel Alegre. Il se sont d'ailleurs considérablement éloignés de la forme traditionnelle du fado au profit d'une chanson plus libre et plus créative sur le plan tant poétique que musical.

C'est dans la lignée de ces deux chanteurs que le fadiste F. Machado Soares se situe luimême, bien qu'il nous semble pourtant être plus classique et plus proche de cette école étudiante et érudite dont nous venons de parler.

Jouant sur de longues séquences mélodiques ponctuées de silences, le fado de Coimbra exprime la langueur plutôt que le sentiment dramatique. Il développe des mélodies très linéaires, auxquelles vient se superposer un accompagnement de caractère horizontal, alors que le fado de Lisbonne présente des mélodies plus découpées, avec des accords de guitare qui rencontrent régulièrement la voix. Le répertore de F. Machado Soares est certes d'une grande musicalité et les guitaristes qui l'accompagnent, José Fontes Rocha et Durval Moreirinhas, se révèlent être de grands interprètes, en particulier dans les longs intermèdes instrumentaux qui caractérisent la tradition de Coimbra. Malheureusement, de bons guitaristes ne font pas de bons fadistes. Sorte de baryton aux gémissements continus, la voix de F. Machado Soares est empreinte d'un affect et d'une emphase tellement excessifs que le chant perd toute sa densité émotive. Pourquoi enrober ses mélodies de tant de volutes inutiles, plus propres au style de l'opéra qu'à celui du fado ? Si la partie instrumentale est remarquablement mélodieuse tout en restant sobre, la prestation chantée confine au ridicule et annonce la dégénérescence du genre. Alors qu'Amalia Rodrigues contient si bien le déversement des sentiments à l'intérieur des mille nuances de sa voix, le lyrisme baroque et sirupeux de F. Machado Soares est à faire frémir les auditeurs les moins avertis.

Quant au texte de la brochure, il s'inscrit exactement dans le même esprit que l'interprétation de la musique. Des commentaires de style éculé viennent entourer le fado d'un halo mystérieux sans apporter aucune information précise sur le vrai contexte d'interprétation de celui-ci, ni sur ses styles. De plus, la traduction française du texte portugais ne restitue qu'en partie ses nuances originelles, ce qui rend parfois le propos confus. Ni historique, ni fresque sociale d'actualité ne viennent nourrir ce disque qui est en outre d'une prétention rare. En effet, ne lit-on pas dans la brochure que le disque que F. Machado Soares a publié précédemment chez Polygram (1986) «est considéré par la critique comme l'un des meilleurs disques de musique portugaise de tous les temps ».

Par ses propos comme par son contenu musical, le disque de la collection Ocora est une insulte aux grands artistes du Portugal, tels les fadistes Amalia Rodrigues, M. T. de Noronha, A. Marceneiro, Manuel Vicente et même Carlos do Carmo... qui pourtant ne fait pas partie des meilleurs. De surcroît, comment F. Machado Soares a-t-il pu s'égarer à ce 
point hors du foyer artistique de Coimbra? Sans doute a-t-il construit son épopée personnelle tout seul, se tenant volontairement à l'abri d'une grande école de musiciens.

Est-ce la raison pour laquelle le texte de la brochure n'est pas signé ? Il est pour le moins surprenant que la prestigieuse collection Ocora de Radio France ait pu tomber sur un si médiocre fadiste. Mais sans doute le Portugal pose-t-il encore des pièges à celui qui ne le pénètre pas et que la simple différence culturelle enchante.

Fig. 3 : Une bergère avec son troupeau

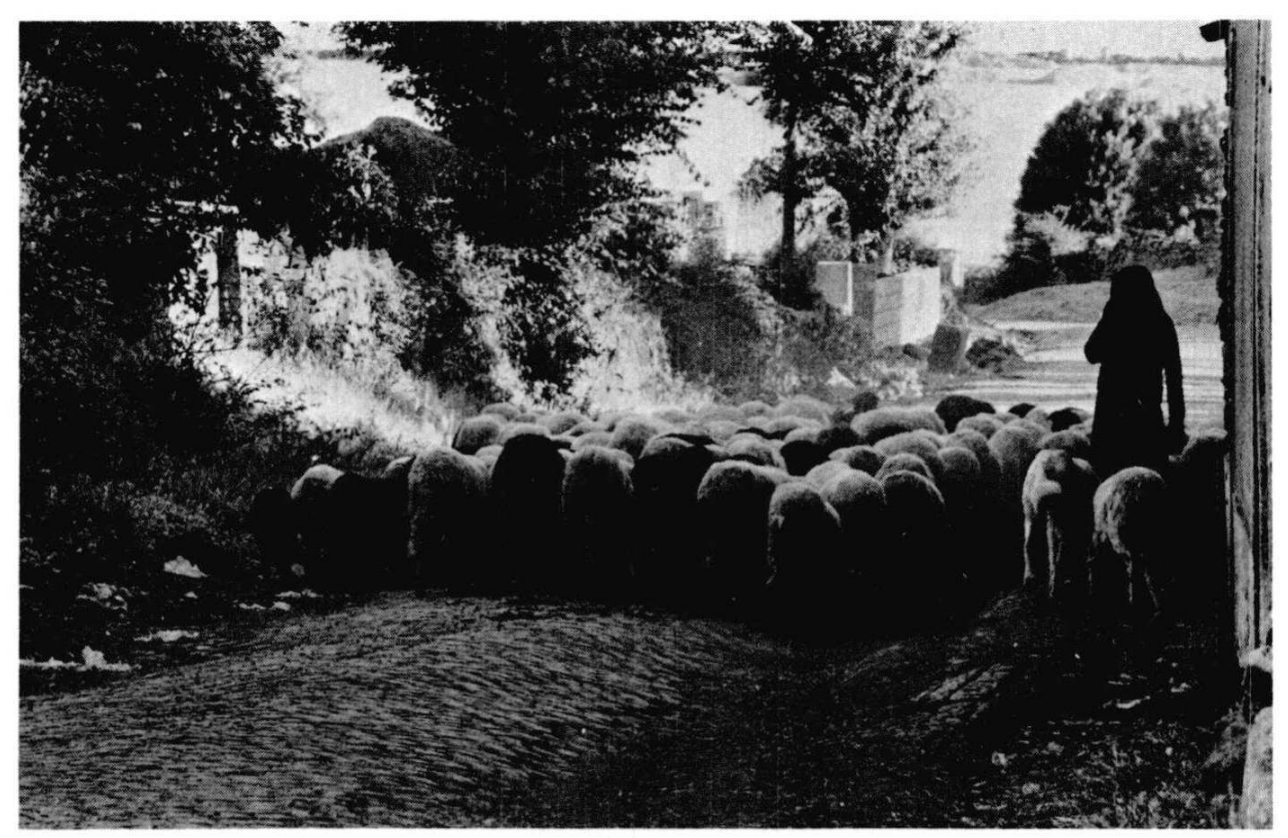

Photo : Anne Caufriez.

Le titre du disque Fado... fados, la célèbre Fernando Maria, édité dans la collection française Arion (Paris 1989), annonce d'emblée un état d'esprit : vendre la musique d'un pays aux touristes. C'est pourquoi il faut rappeler que le fado est une chanson mélodramatique née dans les quartiers populaires de Lisbonne.

31 Aujourd'hui, il y a le fado des milieux pauvres et celui des milieux « cultivés ». C'est à la première catégorie que se rattache le talent de Fernanda Maria à laquelle ce disque est principalement consacré et qui incarne personnellement les bas quartiers dont elle chante l'infortune. Fadiste populaire très connue, sa réputation n'avait guère franchi les frontières du Portugal avant la sortie de cette publication française. Cela fait pourtant plus de vingt ans qu'elle a publié ses premiers disques à Lisbonne. Sa voix exprime très bien la compassion de la saudade, ce sentiment de tristesse si cher au fado, que vient appesantir le port habituel d'un grand châle noir.

Si Fernanda Maria traduit de façon plus anecdotique et plus affectée que d'autres cet état d'âme, c'est parce qu'elle incarne le peuple, celui des bars et des tavernes du vieux Lisbonne qui vit toujours dans une certaine pauvreté. Contrairement à d'autres fadistes qui s'inspirent de textes déjà composés ou qui chantent les grands poètes portugais (telle Amalia Rodrigues), les paroles des fados de F. Maria ont gardé toutes les images et les sous-entendus du langage populaire, faits à la fois d'allusions corsées et de déversements touchants. Cette artiste lisboète par excellence est ici accompagnée de deux guitaristes 
dont le disque ne mentionne pas le nom. Leur jeu mélodieux et honnête ne présente toutefois pas la finesse des instrumentistes. La voix de F. Maria qui exprime si bien l'affliction ou la douleur sans rémission dans les fados les plus tristes et l'envol des sentiments d'amour dans les fados les plus alertes aurait suffi à faire un bon disque. Mais l'addition d'autres interprètes qui semblent ici protégés par sa notoriété, n'apportent rien à cette publication. Ils viennent au contraire l'affaiblir.

En effet, le titre du disque nous livre les noms de trois fadistes supplémentaires, MariaGloria Guedes, Lucinda Sobral et Helder Antonio. De ces fadistes de second plan, on ne retiendra que celui de la plage 7, qui est probablement Helder Antonio, car les autres exécutent le fado plus qu'ils ne l'interprètent. Tel est le cas des deux femmes dont les voix semblent linéaires et insipides comparées à la voix fervente de F. Maria. C'est précisément là que se situe le talent de cette célèbre fadiste, qui réside dans sa capacité de mettre en scène les situations qu'elle interprète, de traduire les accents dramatiques du texte, de moduler la mélodie dans les registres mineurs pour imprégner le chant de toute la mélancolie qui en est l'âme.

Quant au texte de la brochure, son grand mérite est de nous plonger immédiatement dans l'esprit du fado par une petite introduction au genre et par la traduction (partielle) des paroles qui remplacent ici les commentaires sur les enregistrements. Ainsi pénètre-t-on d'emblée la poétique amoureuse du fado ou les sentiments d'un milieu social modeste porté vers la résignation et l'alcool. Car le fado, exprimant la tristesse du destin, appartient aux pouvoirs occultes du fatalisme...

En revanche, ce texte contient plusieurs erreurs, quand il ne tombe pas dans des clichés qui n'ont rien à voir avec les réalités de cette chanson. Contrairement à ce qui y est affirmé, le fado est un genre pratiqué avant 1840 et les guitares du fado (qui sont bien particulières) ne sont pas celles qui accompagnent le paysan ou l'ouvrier dans la vie quotidienne, sinon exceptionnellement. En effet, les régions paysannes qui ont conservé la tradition de la guitare présentent des instruments bien différents. En outre, Severa (plage 18) n'est pas «le plus fameux chanteur de fado qui ait jamais existé », mais bien la chanteuse de fado la plus célèbre de Lisbonne, morte en 1845 à l'âge de 26 ans. La qualité de sa voix et l'amour que lui porta le comte de Vimioso en ont fait un véritable mythe national. C'est probablement aussi l'ignorance du sujet qui a amené l'éditeur du disque à accepter l'addition d'instruments populaires qui sont complètement étrangers au genre du fado, tels l'accordéon et les triangles percutés ferrinhos venant se superposer au jeu des guitares (plages 1 et 6). Cet artifice ne manque pas de ridicule si l'on sait que le fado est basé sur le jeu unique de deux guitares, invariablement les mêmes: la "guitare portugaise » et la guitare classique. Le texte semble aussi confondre les quartiers de Lisbonne, car les musiciens y sont présentés comme artistes de la Mauraria qui est l'ancien quartier maure de la ville. Précisons d'emblée que les fadistes ont déserté depuis longtemps la Mauraria au profit de l'Alfama et de Bairo Alto, devenus les véritables lieux d'interprétation de cette chanson. C'est en fait en 1893 que le fado a été cantonné dans la Mauraria, le pouvoir libéral de l'époque désirant limiter sa propagation à travers la ville. Entretemps, la situation a bien changé...

L'intérêt de ce disque inégal réside principalement dans la révélation d'une veine de fados populaires qui, d'habitude, restent prisonniers des murs de la taverne. Mais le Portugal a déjà publié plus d'un disque sur le fado des quartiers pauvres. A cet égard, le disque Arion n'apporte rien de neuf, d'autant que la voix de F. Maria a perdu beaucoup de sa force 
émotionnelle et de sa conviction pathétique par rapport aux disques qu'elle a publiés il y a vingt ans à Lisbonne.

\section{NOTES}

1. $O$ outro Alentejo. [J.A. Sardinha]. Recolhas musicais da tradiçao oral, colecçao « Roseira Brava ». Contradança LP 86-02, Lisboa 1986.

2. H. de Fraysseix est bien connu pour les disques qu'il a publiés dans la collection de l'UNESCO. V. Pereira est un des pionniers de l'ethnomusicologie au Portugal. Professeur au Conservatoire de Porto dans les années 1940-50, il a collecté la musique traditionnelle de plusieurs provinces du pays. Il est ici l'auteur des enregistrements des plages 7, 15, 16 et 17.

3. Il s'agit ici d'un célèbre musicien du village de Idanha-A-Nova (Beira Baixa), José dos Reis. 